\title{
Direct Access to Ketones from Aldehydes via Rhodium-Catalyzed Cross- Coupling Reaction with Potassium Trifluoro(organo)borates
}

\author{
Mathieu Pucheault, Sylvain Darses, ${ }^{*}$ Jean-Pierre Genet ${ }^{\star}$ \\ Laboratoire de Synthèse Sélective Organique (UMR 7573, CNRS), Ecole Nationale Supérieure de Chimie \\ de Paris, 11 rue P\&M Curie, 75231 Paris cedex 05 (France)
}

\section{Supporting Information}

\section{General Procedures}

${ }^{1} \mathrm{H}$ NMR and ${ }^{13} \mathrm{C}$ NMR spectra were recorded on a Avance 300 or Avance 400 instruments; chemical shifts $(\delta)$ are reported in ppm relative to $\mathrm{Me}_{4} \mathrm{Si}$; coupling constants $(J)$ are reported in $\mathrm{Hz}$ and refer to apparent peak multiplicities. ${ }^{2} \mathrm{H}$ NMR spectra were recorded on a Bruker Avance 400 instrument at $61.4 \mathrm{MHz}$ using benzene- $d_{6}$ as an internal reference. Mass spectra were determined on a Ribermag instrument. High resolution mass spectra were performed on a Varian MAT311 at the Université Pierre et Marie Curie (Paris). Thin layer chromatography was carried out on silica-gel plates (Merck F254), spots were detected with UV light and revealed with KMnO4 solution. GC analyses were performed on a Hewlett-Packard instrument equipped with a J\&W Scientific HP-01 capillary column $(30 \mathrm{~m}, \varnothing=0.25 \mu \mathrm{m})$, using an ionization flame detector.

Potassium organotrifluoroborates ${ }^{1}$ were prepared according to published procedures. Dioxane and acetone were freshly distilled from $\mathrm{CaH}_{2}$.

\section{Formation of deuteriated compounds.}

2-deuteriopropan-2-ol, 1-deuteriodiphenylmethanol and 1-deuteriophenylmethanol were prepared by $\mathrm{NaBD}_{4}$ reduction of acetone, benzophenone and benzaldehyde respectively.

2-deuteriopropan-2-ol: ${ }^{2} \mathrm{H} \mathrm{NMR}\left(\mathrm{CDCl}_{3}, 300 \mathrm{MHz}\right) \delta=0.95\left(\mathrm{t}, J_{\mathrm{D}-\mathrm{H}}=7.0 \mathrm{~Hz}, 6 \mathrm{H}\right), 4.53(\mathrm{~s}, 1 \mathrm{H}) .{ }^{2} \mathrm{H} \mathrm{NMR}$ $\left(\mathrm{CDCl}_{3}, 61.4 \mathrm{MHz}\right) \delta=3.75$ (hept, $\left.J_{\mathrm{D}-\mathrm{H}}=0.8 \mathrm{~Hz}\right) .{ }^{13} \mathrm{C} \mathrm{NMR}\left(\mathrm{CDCl}_{3}, 75 \mathrm{MHz}\right) \delta=24.6,63.0\left(\mathrm{t}, J_{\mathrm{D}-\mathrm{C}}=21.4 \mathrm{~Hz}\right)$.

1-deuteriodiphenylmethanol: ${ }^{3} \mathrm{H}$ NMR $\left(\mathrm{CDCl}_{3}, 300 \mathrm{MHz}\right) \delta=2.65$ (br s, 1H), 7.25-7.42 (m, 10H). ${ }^{2} \mathrm{H} \mathrm{NMR}$ $\left(\mathrm{CDCl}_{3}, 61.4 \mathrm{MHz}\right) \delta=5.52(\mathrm{~s}) .{ }^{13} \mathrm{C} \mathrm{NMR}\left(\mathrm{CDCl}_{3}, 75 \mathrm{MHz}\right) \delta=75.9\left(\mathrm{t}, J_{\mathrm{D}-\mathrm{C}}=21.4 \mathrm{~Hz}\right), 126.8,127.7,128.7$, 144.0 .

1-deuteriophenylmethanol: ${ }^{4} \mathrm{H}$ NMR $\left(\mathrm{CDCl}_{3}, 300 \mathrm{MHz}\right) \delta=3.6$ (br s, 1H), 4.57 (br s, 1H), 7.25-7.33 (m, 5H). ${ }^{2} \mathrm{H} \mathrm{NMR}\left(\mathrm{CDCl}_{3}, 61.4 \mathrm{MHz}\right) \delta=4.34\left(\mathrm{~d}, J_{\mathrm{D}-\mathrm{H}}=2.0 \mathrm{~Hz}\right) .{ }^{13} \mathrm{C} \mathrm{NMR}\left(\mathrm{CDCl}_{3}, 75 \mathrm{MHz}\right) \delta=64.4\left(\mathrm{~d}, J_{\mathrm{D}-\mathrm{C}}=22 \mathrm{~Hz}\right)$, 127.0, 127.4, 128.4, 141.1.

Typical procedure (conditions B) for carbonylation of potassium trifluoro(organo)borates: preparation of (4-hydroxy-3-methoxyphenyl)-2-methylphenylmethanone (entry 8):

To a mixture of potassium trifluoro(2-methylphenyl)borate 2 d (1 mmol, $198 \mathrm{mg}$ ), 4-hydroxy-3methoxybenzaldehyde $\mathbf{1 h}(0.5 \mathrm{mmol}, 76 \mathrm{mg})$ and chlorobis(ethylene)rhodium(I) dimer $(7.5 \mu \mathrm{mol}, 2.9 \mathrm{mg})$ in degassed dioxane $(2 \mathrm{~mL})$ was added a solution of tri-tert-butylphosphane in degassed toluene $(15 \mu \mathrm{mol}, 3 \mathrm{M}$ solution, $50 \mu \mathrm{L}$ ), under an argon atmosphere. After 5 minutes stirring at room temperature, degassed acetone $(0.5 \mathrm{~mL})$ was added and the flask was placed in a preheated oil bath at $80^{\circ} \mathrm{C}$. The mixture was stirred until completion of the reaction (followed by GC analysis). After concentration under reduced pressure, the crude mixture was purified by silica gel chromatography eluting with cyclohexane/ethyl acetate (4:1) to afford $141 \mathrm{mg}$ of analytically pure ketone as a pale yellow oil (yield 94\%).

\footnotetext{
${ }^{1}$ a) Darses, S.; Genet, J.-P. Eur. J. Org. Chem. 2003, 4313. (b) Darses, S.; Michaud, G.; Genet, J.-P. Eur. J. Org. Chem. 1999, 1875.

${ }^{2}$ Yamashita, M.; Dai, F.-Y.; Suzuki, M.; Saito, Y. Bull. Chem. Soc. Jpn. 1991, 64, 628.

${ }^{3}$ Alonso, F.; Vitale, C.; Radivoy, G.; Yus, M. Synthesis 2003, 443.

${ }^{4}$ Kim, J.-M.; Bogdan, M. A.; Mariano, P. S. J. Am. Chem. Soc. 1993, 115, 10591.
} 
GC (HP-01, $30 \mathrm{~m}, 4 \mathrm{~mL} / \mathrm{min}$., $70^{\circ} \mathrm{C}$ during $1 \mathrm{~min}$. then $20^{\circ} \mathrm{C} / \mathrm{min}$ up to $250^{\circ} \mathrm{C}$ ): $\mathrm{T}_{\mathrm{R}} 9.21 \mathrm{~min}$. $\mathrm{Rf}$ (cyclohexane/AcOEt 4/1): 0.23. ${ }^{1} \mathrm{H} \mathrm{NMR}\left(\mathrm{CDCl}_{3}, 300 \mathrm{MHz}\right) \delta=2.29(\mathrm{~s}, 3 \mathrm{H}), 3.93(\mathrm{~s}, 3 \mathrm{H}), 6.33(\mathrm{~s}, 1 \mathrm{H}), 6.87(\mathrm{~d}$, $J=8.5 \mathrm{~Hz}, 1 \mathrm{H}), 7.18(\mathrm{dd}, J=1.9 \mathrm{~Hz}, J=8.5 \mathrm{~Hz}, 1 \mathrm{H}), 7.20-7.34(\mathrm{~m}, 4 \mathrm{H}), 7.58(\mathrm{~d}, J=1.9 \mathrm{~Hz}, 1 \mathrm{H}) .{ }^{13} \mathrm{C} \mathrm{NMR}$ $\left(\mathrm{CDCl}_{3}, 75 \mathrm{MHz}\right) \delta=19.7,56.1,110.9,113.8,125.1,126.8,127.8,129.8,130.3,130.8,136.1,139.1,146.8$, 150.7, 197.5. MS (70 eV): m/z (\%) = $242(91)[\mathrm{M}]^{+\bullet}, 225(62), 211$ (82), 151 (67), 119 (37), 91 (100). HRMS for $\mathrm{C}_{15} \mathrm{H}_{14} \mathrm{O}_{3}$ calculated 242.0943; found 242.0950.

\section{(3-trifluoromethylphenyl)-2-thienylmethanone (entry 7):}

Reaction of potassium 3-trifluoromethylphenyltrifluoroborate $\mathbf{2}$ c $\left(\begin{array}{lllll}1 & \mathrm{mmol}, & 252 & \mathrm{mg}\end{array}\right)$ and 2-thiophenecarboxaldehyde $\mathbf{1 g}(0.5 \mathrm{mmol}, 56 \mathrm{mg})$ afforded $106 \mathrm{mg}$ of ketone as a colorless oil (yield 83\%). GC ( $4 \mathrm{~mL} / \mathrm{min}, 70^{\circ} \mathrm{C}$ during $1 \mathrm{~min}$ then $20^{\circ} \mathrm{C} / \mathrm{min}$ up to $250^{\circ} \mathrm{C}$ ): $\mathrm{T}_{\mathrm{R}} 6.94 \mathrm{~min}$. Rf (cyclohexane/ ethyl acetate $4 / 1$ ): 0.34. ${ }^{1} \mathrm{H} \mathrm{NMR}\left(\mathrm{CDCl}_{3}, 300 \mathrm{MHz}\right) \delta=7.19(\mathrm{dd}, J=3.9 \mathrm{~Hz}, J=5.0 \mathrm{~Hz}, 1 \mathrm{H}), 7.61(\mathrm{dd}, J=1.1 \mathrm{~Hz}, J=5.0 \mathrm{~Hz}$, $1 \mathrm{H}), 7.64(\mathrm{t}, J=7.8 \mathrm{~Hz}, 1 \mathrm{H}), 7.77(\mathrm{dd}, J=3.9 \mathrm{~Hz}, J=1.1 \mathrm{~Hz}, 1 \mathrm{H}), 7.84(\mathrm{dd}, J=0.5 \mathrm{~Hz}, J=7.8 \mathrm{~Hz}, 1 \mathrm{H}), 8.03$ $(\mathrm{dd}, J=0.5 \mathrm{~Hz}, J=7.8 \mathrm{~Hz}, 1 \mathrm{H}), 8.11(\mathrm{~s}, 1 \mathrm{H}) .{ }^{13} \mathrm{C} \mathrm{NMR}\left(\mathrm{CDCl}_{3}, 75 \mathrm{MHz}\right) \delta=123.6\left(\mathrm{q}, J_{\mathrm{C}-\mathrm{F}}=270.9 \mathrm{~Hz}\right), 125.9$ $\left(\mathrm{q}, J_{\mathrm{C}-\mathrm{F}}=3.7 \mathrm{~Hz}\right), 128.3,128.67,128.7,129.1,131.0\left(\mathrm{q}, J_{\mathrm{C}-\mathrm{F}}=32.7 \mathrm{~Hz}\right), 132.2,135.0\left(\mathrm{q}, J_{\mathrm{C}-\mathrm{F}}=6.4 \mathrm{~Hz}\right), 138.8$, 142.9, 186.7. MS (70 eV): m/z $(\%)=256(38)\left[\mathrm{M}^{+}, 173(10), 145\right.$ (18), 111 (100). HRMS for $\mathrm{C}_{12} \mathrm{H}_{7} \mathrm{~F}_{3} \mathrm{OS}$ calculated 256.0170; found 256.0174 .

\section{(3-bromophenyl)-2-furylmethanone (entry 6):}

Reaction of potassium 3-bromophenyltrifluoroborate $\mathbf{2 b}(1 \mathrm{mmol}, 262 \mathrm{mg})$ and 2-furfural $\mathbf{1 f}(0.5 \mathrm{mmol}, 48 \mathrm{mg})$ afforded $114 \mathrm{mg}$ of ketone as a pale yellow oil (yield $91 \%)$. GC $\left(4 \mathrm{~mL} / \mathrm{min}, 70^{\circ} \mathrm{C}\right.$ during $1 \mathrm{~min}$ then $20^{\circ} \mathrm{C} / \mathrm{min}$ up to $\left.250^{\circ} \mathrm{C}\right): \mathrm{T}_{\mathrm{R}} 7.71 \mathrm{~min}$. Rf (cyclohexane/ ethyl acetate 4/1): 0.27. ${ }^{1} \mathrm{H}$ NMR $\left(\mathrm{CDCl}_{3}, 300 \mathrm{MHz}\right) \delta=6.53(\mathrm{dd}, J=$ $3.6 \mathrm{~Hz}, J=1.9 \mathrm{~Hz}, 1 \mathrm{H}), 7.15(\mathrm{dd}, J=3.6 \mathrm{~Hz}, J=0.8 \mathrm{~Hz}, 1 \mathrm{H}), 7.25(\mathrm{dt}, J=0.4 \mathrm{~Hz}, J=7.8 \mathrm{~Hz}, 1 \mathrm{H}), 7.57-7.61$ $(\mathrm{m}, 2 \mathrm{H}), 7.79(\mathrm{ddd}, J=1.2 \mathrm{~Hz}, J=1.5 \mathrm{~Hz}, J=7.8 \mathrm{~Hz}, 1 \mathrm{H}), 7.99(\mathrm{dt}, J=0.8 \mathrm{~Hz}, J=2.1 \mathrm{~Hz}, 1 \mathrm{H}) .{ }^{13} \mathrm{C} \mathrm{NMR}$ $\left(\mathrm{CDCl}_{3}, 75 \mathrm{MHz}\right) \delta=112.4,120.9,122.6,127.8,130.0,132.2,135.4,138.9,147.4,151.9,180.7 . \mathrm{MS}(70 \mathrm{eV})$ : $\mathrm{m} / \mathrm{z}(\%)=250(2)[\mathrm{M}]^{+}, 183$ (15), 171 (13), 95 (100). HRMS for $\mathrm{C}_{11} \mathrm{H}_{7} \mathrm{BrO}_{2}$ calculated 249.9629; found 249.9361 .

All other products were identified by comparison of spectroscopic data with authentic sample or with those reported in the literature: diphenylmethanone [119-61-9], (4-methoxyphenyl)phenylmethanone [611-94-9], (2methoxyphenyl)phenylmethanone [2553-04-0], (4-hydroxyphenyl)phenylmethanone [1137-42-4], (3hydroxyphenyl)phenylmethanone [117-99-7], (4-fluorophenyl)phenylmethanone [345-83-5], 2furylphenylmethanone $^{5}$ and (4-hydroxyphenyl)-2,4,6-trimethylphenylmethanone ${ }^{6}$ and $1-(4-$ methoxyphenyl)propan-1-one. ${ }^{7}$

\footnotetext{
${ }^{5}$ GooBen, L. J.; Winkel, L.; Döhring, A.; Gosh, K.; Paetzold, J. Synlett 2002, 1237.

${ }^{6}$ Syamala, M. S.; Nageswer Rao, B.; Ramamurthy, V. Tetrahedron 1988, 44, 7234.

${ }^{7}$ Jung, M. E.; Lee, B. S. J. Org. Chem. 2000, 65, 9241.
} 